1,000. Even fewer pig sequences were collected, with one-third of the countries that are home to more than 4 million pigs depositing none at all.

The size of a country's poultry population is no predictor of how many samples that country will generate (see 'Many birds, few samples'). Countries that have welldeveloped veterinary services and a wellstructured and hygienic farming industry inevitably have fewer flu sequences to report, as disease levels tend to be low, says Brown. However, many of the countries that have contributed few or no sequences have poor veterinary systems and flu-prone farming systems, such as backyard farms and mixed poultry and pig farms, which are often close to wild ducks and other flu reservoirs.

"Proper geographic representation is lacking," says van der Werf, as is sustained surveillance. This results in large gaps in data, she says, because "many consecutive years of surveillance are needed to see trends" (see page 535). Poorer countries tend to have inadequate surveillance resources, and farmers often have little incentive to report outbreaks because they will not receive any compensation for culled livestock. Countries sometimes also fail to look for, or report, outbreaks so that they can claim they are free of infection and so avoid trade problems.

Flu experts say that the dire state of surveillance could be rapidly turned around by, for example, creating a network of sentinel sites, focusing on the countries and regions most at risk, that would collect isolates and sequence them in real time. Such a network would probably even cost less than the fragmented and uncoordinated surveillance efforts in place today, says Jeremy Farrar, director of the Oxford University Clinical Research Unit in Ho Chi Minh City, Vietnam (see page 534).

The problem is that no global body has overall responsibility for flu surveillance. The World Health Organization (WHO) runs a global network of labs for human flu surveillance and selects human strains to be included in vaccines for seasonal flu. Monitoring animals falls to the FAO, which tends to focus on food security, and the OIE, which looks mostly at animal health and trade.

What is needed is international leadership, says Farrar. "If, say, the WHO and the FAO were to construct an advisory framework, surveillance could probably be done much more systematically and efficiently." - SEE EDITORIALP. 509

1. Butler, D. \& Ruttimann, J. Nature 441, 137-139 (2006)

2. Check, E. Nature 442, 348-350 (2006).

3. Butler, D. Nature http://dx.doi.org/10.1038/ news050801-1 (2005).

4. Butler. D. Nature http://dx.doi.org/10.1038/ news060206-7 (2006)

5. Nature 440, 255-256 (2006).

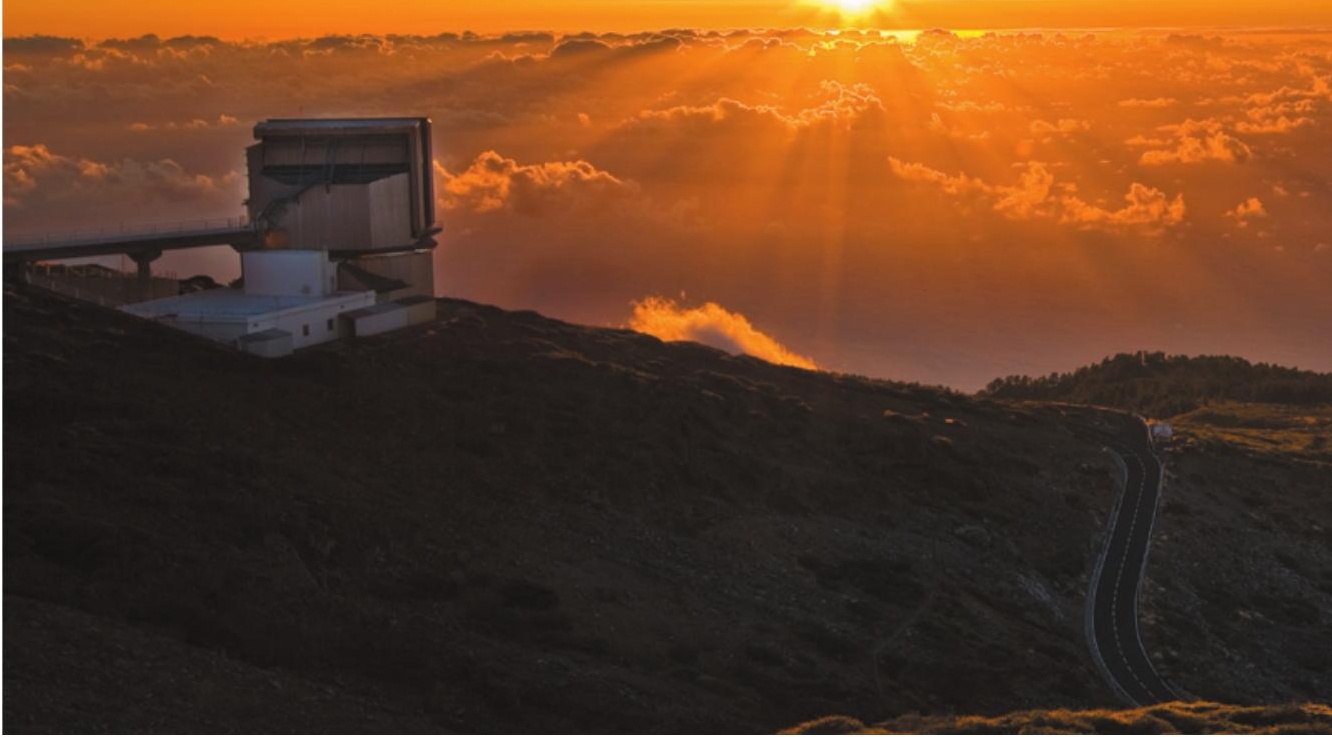

Italy's National Galileo Telescope in the Canary Islands will host the HARPS-North planet finder.

SPACE

\title{
North set for mass analysis of planets
}

\section{Spectrograph will review results from Kepler telescope.}

\section{BY ERIC HAND}

$\mathrm{H}$ ow many extrasolar planets has NASA's Kepler mission discovered? That depends on how you count. Last month, the mission team published a catalogue that lists a staggering 2,321 candidate planets, amassed since May 2009 as the space-based telescope watches stars for the shadow of planets passing over their faces. Yet only 69 of them are considered confirmed planets. Astronomers have fretted over the growing backlog, but help is on its way.

For a Kepler planet to ascend from candidate to confirmed, a second method has to vouch for it: for example, a ground-based spectrograph must report signs that the planet's gravity is tugging its star back and forth. Yet Kepler looks north, whereas the instrument most sensitive to stellar wobbles, the European Southern Observatory's High Accuracy Radial Velocity Planet Searcher (HARPS), is located at the La Silla Observatory in Chile and can only observe the southern sky. On 1 April, however, the Northern Hemisphere will get a near-clone of HARPS when HARPS-North achieves first light at the Italian 3.6-metre National Galileo Telescope (TNG) on La Palma in the Canary Islands.

The instrument has been a long time coming. Conceived in 2005, the project was originally led by Harvard University in Cambridge, Massachusetts. But in 2010, after Harvard's endowment fell during the financial crisis, the University of Geneva in Switzerland took charge. Financial problems forced the group to switch from the 4.2-metre William Herschel Telescope, also on La Palma, to the TNG, which will give the HARPS-North team 80 nights of dedicated time per year for five years.

That should help to alleviate the bottleneck for Kepler candidates. Many astronomers, however, are looking to HARPS-North less for confirmation of the candidate planets than for insight into their properties. The false-positive rate for Kepler, after all, has already been shown to be less than $10 \%$ (T. D. Morton and J. A. Johnson Astrophys. J. (in the press) Preprint at http://arxiv.org/ abs/1101.5630; 2011). "It has become acceptable to do a statistical analysis and say, 'They

ONATURE.COM For more on Kepler's search for exoplanets, visit: go.nature.com/ptghht are planets'," says Joshua Winn, an astronomer at the Massachusetts Institute of Technology in Cambridge.

What Winn and 
others want to know is: what kind of planets? The stellar dimming that Kepler detects is a function of the planet's size. The stellar wobble seen by instruments such as HARPS-North, by contrast, reveals a planet's mass. With knowledge of both mass and size, astronomers can compute the planet's density, which is the key to understanding the nature of the superEarths - planets a few times the size of Earth (see 'Sizing up the candidates') - that are emerging from Kepler's discoveries. "You really want to know if it's a large Earth or a miniNeptune or something different altogether, like a ball of water," says David Charbonneau, a Harvard astronomer and a collaborator on the HARPS-North project.

Until now, Kepler has relied for follow-up observations on an instrument on one of the twin 10-metre telescopes at the Keck Observatory atop Mauna Kea in Hawaii, which can detect a stellar wobble of about 150 centimetres per second - barely good enough to confirm super-Earths. HARPS achieves twice that sensitivity, and Francesco Pepe, principal investigator of HARPS-North at the University of Geneva, hopes that his project will do the same.

Kepler's ultimate goal is to detect Earthsized planets in Earth-like orbits, and for now, such true Earth analogues remain out of reach of ground-based detectors. (The Sun's wobble owing to Earth is about 9 centimetres

\section{SIZING UP THE CANDIDATES}

Most of the 2,321 planet candidates found by NASA's Kepler space telescope are only a little larger

than Earth. Further observations could show whether these super-Earths are rocky, gassy or watery.

24.6 Earth-size

(Less than 1.25 Earth radii)

O 676 Super-Earth-size

(1.25-2 Earth radii)

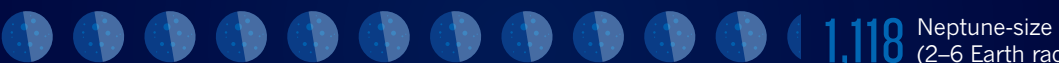

$\because 210$ Jupiter-size

(6-15 Earth radii)

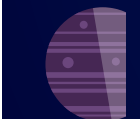

1 Larger

(More than 15 Earth radii)

per second.) To achieve that sort of precision, astronomers want to fit ground-based spectrographs with high-frequency lasers that emit extremely short pulses of light at specific wavelengths spaced equally on the spectrum. These 'laser frequency combs' provide a way to calibrate a spectrograph and synch it to an atomic clock, which eliminates the error resulting from long-term drift of the spectrograph and could offer precision as good as one centimetre per second.

Ronald Walsworth, a physicist at Harvard, plans to take his group's laser comb to HARPS-North later this year. A rival group at the European Southern Observatory is already testing a comb at HARPS.

But these efforts may be for nought. The roiling surfaces of stars jitter, creating a confounding noise in the measurement. It may be that only a small fraction of stars are calm enough for an Earth analogue to be discernible, says Walsworth. "Our future is in the stars," he says. "We'll do the science that the stars enable us to do." 